\title{
Some Conclusions on the Direct Product of Uninorms on Bounded Lattices
}

\author{
${ }^{*}$ Emel Aşıcı ${ }^{a *}$ and Radko Mesiar ${ }^{b, c}$ \\ ${ }^{a}$ Department of Software Engineering, Faculty of Technology, Karadeniz Technical University, \\ 61830 Trabzon, Turkey emelkal in@hotmail.com \\ ${ }^{b}$ Department of Mathematics and Descriptive Geometry, Faculty of Civil Engineering, \\ Slovak University of Technology, Radlinského, 11, 81005 Bratislava, Slovakia \\ mesiar@math.sk \\ ${ }^{c}$ Centre of Excellence IT4Innovations, Division University of Ostrava, \\ IRAFM, 30. Dubna 22, 70103 Ostrava, Czech Republic
}

\begin{abstract}
Recently, the notation of the order induced by uninorms (t-norms, nullnorms) has been studied widely. In this paper, we study on the direct product of uninorms on bounded lattices. Also, we define an order induced by uninorms which are a direct product of two uninorms on bounded lattices. Also, we investigate properties of introduced order.
\end{abstract}

Keywords: Uninorm; direct product; partial order

\section{Introduction}

Aggregation operators [21] play an important role in theories of fuzzy sets, fuzzy logic, fuzzy system modeling, expert systems, neural networks and approximate reasoning $[16,17,21,22,29,33]$. Two basic types of aggregation functions, namely t-norms and tconorms, were introduced by Schweizer and Sklar in [31].

Uninorms with the neutral element $e$ on the unit interval $[0,1]$, as an important generalization of t-norms and $t$-conorms. In contrast to the definitions of t-norms and $\mathrm{t}$-conorms, the difference is that uninorms allow the neutral element $e$ lying anywhere in the unit interval $[0,1]$. In particular, a uninorm $U$ is a t-norm $T$ and t-conorm $S$ when the case $e=1$ and $e=0$, respectively.

As a generalization of t-norms and t-conorms, Yager and Rybalov [32] introduced the concepts of uninorms, then Fodor et al. [19, 20] systematically studied them which are special aggregation functions with the neutral element $e \in[0,1]$. For uninorms on bounded lattices, there also arises much work. First, uninorms on bounded lattices were introduced by Karaçal and Mesiar [25] in 2015. They showed the existence of uni-

\footnotetext{
${ }^{*}$ Corresponding author
}

norms with neutral element $e$ for an arbitrary element $e \in L \backslash\{0,1\}$ with underlying t-norms and t-conorms on an arbitrary bounded lattice. Also, they introduced the smallest and the greatest uninorm with the neutral element $e \in L \backslash\{0,1\}$.

In [14], direct product of triangular norms on product lattices was introduced and some of the algebraic properties were investigated.

In [27], $T$ - partial order, denoted $\preceq_{T}$, defined by means of t-norms on a bounded lattice was introduced. Based on this study, in $[18,7] U$-partial order, denoted $\preceq_{U}$ and $F$-partial order, denoted by $\preceq_{F}$ defined by means of uninorms and nullnorms, respectively.

In this paper, we study on the direct product of uninorms on bounded lattices. The present paper consists of four main parts. Firstly, we give in preliminaries some necessary definitions we will work with. In Section 3, we define an order induced by uninorms which are a direct product of two uninorms on bounded lattices. In Section 4, we define the set of comparable elements with respect to the $U$-partial order and we obtain some interesting results related to direct product of uninorms on $[0,1]^{2}$. In Section 5, some concluding remarks are added.

\section{Preliminaries}

A lattice is a partially ordered set $(L, \leq)$ in which each two element subset $\{x, y\}$ has an infimum, denoted as $x \wedge y$, and a supremum, denoted as $x \vee y$.

A bounded lattice $(L, \leq, 0,1)$ is a lattice that has the bottom and top elements written as 0 and 1 , respectively.

Given a bounded lattice $(L, \leq, 0,1)$ and $a, b \in L$, if $a$ and $b$ are incomparable, in this case, we use the notation $a \| b($ see $[4,5,6,9,12,23,26])$.

Definition 1 [14]Let $\quad\left(L_{1}, \leq_{1}, 0_{1}, 1_{1}\right)$
$\left(L_{2}, \leq_{2}, 0_{2}, 1_{2}\right)$ be bounded lattices. 
$L_{1} \times L_{2}=\left(L_{1} \times L_{2}, \leq,\left(0_{1}, 0_{2}\right),\left(1_{1}, 1_{2}\right)\right)$ is a bounded lattice with partial order relation $\leq, \wedge$ and $\vee$ defined by

$$
\begin{gathered}
\left(x_{1}, y_{1}\right) \leq\left(x_{2}, y_{2}\right) \Leftrightarrow x_{1} \leq_{1} x_{2} \text { and } y_{1} \leq_{2} y_{2} . \\
\left(x_{1}, y_{1}\right) \wedge\left(x_{2}, y_{2}\right)=\left(x_{1} \wedge_{1} x_{2}, y_{1} \wedge_{2} y_{2}\right) . \\
\left(x_{1}, y_{1}\right) \vee\left(x_{2}, y_{2}\right)=\left(x_{1} \vee_{1} x_{2}, y_{1} \vee_{2} y_{2}\right) .
\end{gathered}
$$

In this study, we use the $L_{1}$ instead of $\left(L_{1}, \leq_{1}, 0_{1}, 1_{1}\right)$, $L_{2}$ instead of $\left(L_{2}, \leq_{2}, 0_{2}, 1_{2}\right)$ and $L_{1} \times L_{2}$ instead of $\left(L_{1} \times L_{2}, \leq, \wedge, \vee,\left(0_{1}, 0_{2}\right),\left(1_{1}, 1_{2}\right)\right)$.

Definition 2 [8, 30] Let L be a bounded lattice. A triangular norm $T$ (briefly $t$-norm) is a binary operation on $L$ that is commutative, associative, monotone and has neutral element 1.

Example 1 [28] The four basic t-norms $T_{M}, T_{P}, T_{L}$ and $T_{D}$ on $[0,1]$ are given by:

$T_{M}(x, y)=\min (x, y)$,

$T_{P}(x, y)=x \times y$,

$T_{L}(x, y)=\max (x+y-1,0)$,

$T_{D}(x, y)= \begin{cases}0 & (x, y) \in[0,1)^{2}, \\ \min (x, y) & \text { otherwise. }\end{cases}$

Definition 3 [2, 30] Let $L$ be a bounded lattice. A triangular conorm $S$ (briefly t-conorm) is a binary operation on $L$ that is commutative, associative, monotone and has neutral element 0.

Example 2 [28] The four basic t-conorms $S_{M}, S_{P}, S_{L}$ and $S_{D}$ on $[0,1]$ are given by:

$S_{M}(x, y)=\max (x, y)$,

$S_{P}(x, y)=x+y-x \times y$,

$S_{L}(x, y)=\min (x+y, 1)$,

$S_{D}(x, y)= \begin{cases}1 & (x, y) \in(0,1]^{2}, \\ \max (x, y) & \text { otherwise. }\end{cases}$

Extremal t-norms $T_{\wedge}$ and $T_{W}$ are defined on a bounded lattice as follows, respectively:

$T_{\wedge}(x, y)=x \wedge y$

$T_{W}(x, y)= \begin{cases}x & \text { if } y=1, \\ y & \text { if } x=1, \\ 0 & \text { otherwise. }\end{cases}$

Similarly, the t-conorms $S_{\vee}$ and $S_{W}$ can be defined.

Especially we have obtained $T_{W}=T_{D}$ and $T_{\wedge}=T_{M}$ for $L=[0,1] \subset R$.

Definition 4 [14] Let $L_{1}$ and $L_{2}$ be bounded lattices and $T_{1}$ and $T_{2}$ be t-norms on $L_{1}$ and $L_{2}$, respectively. Then, the direct product $T_{1} \times T_{2}$ of $T_{1}$ and $T_{2}$, defined by

$$
T_{1} \times T_{2}\left(\left(x_{1}, y_{1}\right),\left(x_{2}, y_{2}\right)\right)=\left(T_{1}\left(x_{1}, x_{2}\right), T_{2}\left(y_{1}, y_{2}\right)\right)
$$

is a t-norm on the product lattice $L_{1} \times L_{2}$.
Definition 5 [11] A t-norm $T$ on $L$ is divisible if the following condition holds:

$\forall x, y \in L \quad$ with $\quad x \leq y \quad$ there is a $\quad z \in L \quad$ such that

$$
x=T(y, z) .
$$

Definition $6[1,10,13]$ Let L be a bounded lattice. An operation $U: L^{2} \rightarrow L$ is called a uninorm on $L$, if it is commutative, associative, monotone and has a neutral element $e \in L$.

We denote by $\mathscr{U}(e)$ the set of all uninorms on $L$ with the neutral element $e \in L$. Also, we denote by $A(e)=$ $L^{2} \backslash\left([0, e]^{2} \cup[e, 1]^{2}\right)$ and $I(U)=\{x \in L \mid U(x, x)=x\}$.

Theorem 1 [19] Let $U:[0,1]^{2} \rightarrow[0,1]$ be a uninorm with neutral element $e \in(0,1)$. Then the sections $x \mapsto$ $U(x, 1)$ and $x \mapsto U(x, 0)$ are continuous in each point except perhaps for $e$ if and only if $U$ is given by one of the following formulas.

(a) If $U(0,1)=0$, then

$$
U(x, y)= \begin{cases}e T\left(\frac{x}{e}, \frac{y}{e}\right) & ,(x, y) \in[0, e]^{2} \\ e+(1-e) S\left(\frac{x-e}{1-e}, \frac{y-e}{1-e}\right) & ,(x, y) \in[e, 1]^{2} \\ \min (x, y) & ,(x, y) \in A(e),\end{cases}
$$

where $T$ is a $t$-norm and $S$ is a $t$-conorm.

(b) If $U(0,1)=1$, then the same structure holds, changing minimum by maximum in $A(e)$.

The class of uninorms as in case (a) will be denoted by $\mathscr{U}_{\text {min }}$ and the class of uninorms as in case (b) by $\mathscr{U}_{\text {max }}$. We will denote a uninorm $U$ in $\mathscr{U}_{\min }$ with underlying t-norm $T$, underlying t-conorm $S$ and neutral element $e$ by $U \equiv\langle T, e, S\rangle_{\min }$ and in a similar way, a uninorm in $\mathscr{U}_{\max }$ by $U \equiv\langle T, e, S\rangle_{\max }$.

Proposition 1 [24] Let $L_{1}$ and $L_{2}$ be bounded lattices and $U_{1}$ be a uninorm on $L_{1}$ with neutral element $e_{1}$ and $U_{2}$ be a uninorm on $L_{2}$ with neutral element $e_{2}$. Then the direct product $U_{1} \times U_{2}$ of $U_{1}$ and $U_{2}$, defined by

$$
U_{1} \times U_{2}\left(\left(x_{1}, y_{1}\right),\left(x_{2}, y_{2}\right)\right)=\left(U_{1}\left(x_{1}, x_{2}\right), U_{2}\left(y_{1}, y_{2}\right)\right)
$$

is a uninorm on the product lattice $L_{1} \times L_{2}$ with neutral element $\left(e_{1}, e_{2}\right)$.

Definition 7 [27] Let $L$ be a bounded lattice, $T$ be a $t$-norm on $L$. The order defined as follows is called a $T$ - partial order (triangular order) for t-norm $T$ :

$$
x \preceq_{T} y: \Leftrightarrow T(\ell, y)=x \text { for some } \ell \in L .
$$

Definition 8 [18] Let $L$ be a bounded lattice, $S$ be a $t$-conorm on L. The order defined as follows is called an $S$ - partial order for $t$-conorm $S$ : 


$$
x \preceq_{S} y: \Leftrightarrow S(\ell, x)=y \text { for some } \ell \in L .
$$

Definition 9 [18] Let $L$ be a bounded lattice and $U$ be a uninorm with neutral element e on $L$. Define the following relation, for $x, y \in L$, as

$$
x \preceq_{U} y: \Leftrightarrow\left\{\begin{array}{r}
\text { if } x, y \in[0, e] \text { and there exist } k \in[0, e] \\
\text { such that } U(y, k)=x \text { or, } \\
\text { if } x, y \in[e, 1] \text { and there exist } \ell \in[e, 1] \\
\text { such that } U(x, \ell)=y \text { or, } \\
\text { if }(x, y) \in L^{*} \text { and } x \leq y .
\end{array}\right.
$$

where $I_{e}=\{x \in L \mid x \| e\}$ and $L^{*}=[0, e] \times[e, 1] \cup$ $[0, e] \times I_{e} \cup[e, 1] \times I_{e} \cup[e, 1] \times[0, e] \cup I_{e} \times[0, e] \cup I_{e} \times$ $[e, 1] \cup I_{e} \times I_{e}$.

Proposition 2 [18] The relation $\preceq_{U}$ defined in (2) is a partial order on $L$.

Note: The partial order $\preceq_{U}$ in (2) is called $U$-partial order on $L$.

Definition 10 [3] Let $L$ be a bounded lattice, $U$ be a uninorm on $L$ and let $K_{U}$ be defined by

$$
\begin{gathered}
K_{U}=\left\{x \in L \mid \text { for some } y \in L,\left[x<y \text { and } x \npreceq_{U} y\right]\right. \text { or } \\
\left.\left[y<x \text { and } y \npreceq_{U} x\right]\right\} .
\end{gathered}
$$

\section{$3 \preceq U_{1} \times U_{2}$-partial order}

In this section, we define an order induced by uninorms which are a direct product of two uninorms on bounded lattices.

Definition 11 Let $L_{1}$ and $L_{2}$ be bounded lattices, $U_{1}$ be a uninorm on $L_{1}$ with neutral element $e_{1}$ and $U_{2}$ be a uninorm on $L_{2}$ with neutral element $e_{2}$ and consider their direct product $U_{1} \times U_{2}$ on $L_{1} \times L_{2}$. Let $\preceq_{U_{1}}$ and $\preceq_{U_{2}}$ are partial orders induced by uninorms $U_{1}$ and $U_{2}$, respectively. Then, the relation $\preceq_{U_{1} \times U_{2}}$ is defined by

$$
(x, y) \preceq_{U_{1} \times U_{2}}(z, t) \Leftrightarrow x \preceq_{U_{1}} z \text { and } y \preceq_{U_{2}} t
$$

for all $(x, y),(z, t) \in L_{1} \times L_{2}$.

Proposition 3 Let $U_{1}$ be a uninorm on $L_{1}$ with neutral element $e_{1}$ and $U_{2}$ be a uninorm on $L_{2}$ with neutral element $e_{2}$ and consider their direct product $U_{1} \times U_{2}$ on $L_{1} \times L_{2}$. Then, the relation $\preceq_{U_{1} \times U_{2}}$ defined in Definition 11 is a partial order on $L_{1} \times L_{2}$.

Proposition 4 Let $L_{1}$ and $L_{2}$ be bounded lattices, $U_{1}$ be a uninorm on $L_{1}$ with neutral element $e_{1}$ and $U_{2}$ be a uninorm on $L_{2}$ with neutral element $e_{2}$ and consider their direct product $U_{1} \times U_{2}$ on $L_{1} \times L_{2}$. Then, $L_{1} \times L_{2}$ is a bounded partially ordered set with respect to the $\preceq_{U_{1} \times U_{2}}$ partial order.

Example 3 Consider the lattice $\left(L_{1}=L_{2}=\right.$ $\{0, m, n, e, p, s, k, t, 1\}, \leq, 0,1)$ given in Fig. 1 and the uninorms $U_{1}$ and $U_{2}$ on $L_{1}=L_{2}$ defined Table 1 and Table 2, respectively.

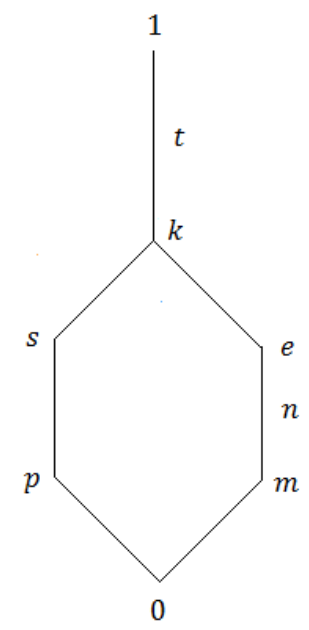

Figure 1: The lattice $L_{1}=L_{2}$

\begin{tabular}{c|ccccccccc}
\multicolumn{10}{c}{ Table 1: The uninorm $U_{1}$ on $L_{1}=L_{2}$} \\
\hline$U_{1}$ & 0 & $m$ & $n$ & $e$ & $p$ & $s$ & $k$ & $t$ & 1 \\
\hline 0 & 0 & 0 & 0 & 0 & $p$ & $s$ & $k$ & $t$ & 1 \\
$m$ & 0 & $m$ & $m$ & $m$ & $p$ & $s$ & $k$ & $t$ & 1 \\
$n$ & 0 & $m$ & $n$ & $n$ & $p$ & $s$ & $k$ & $t$ & 1 \\
$e$ & 0 & $m$ & $n$ & $e$ & $p$ & $s$ & $k$ & $t$ & 1 \\
$p$ & $p$ & $p$ & $p$ & $p$ & 1 & 1 & 1 & 1 & 1 \\
$s$ & $s$ & $s$ & $s$ & $s$ & 1 & 1 & 1 & 1 & 1 \\
$k$ & $k$ & $k$ & $k$ & $k$ & 1 & 1 & 1 & 1 & 1 \\
$t$ & $t$ & $t$ & $t$ & $t$ & 1 & 1 & 1 & 1 & 1 \\
1 & 1 & 1 & 1 & 1 & 1 & 1 & 1 & 1 & 1 \\
\hline
\end{tabular}

Since $U_{1}(m, n)=m$ and $U_{2}(m, n)=m$, then we obtain that $m \preceq_{U_{1}} n$ and $m \preceq_{U_{2}} n$. So, it is obtained $(m, m) \preceq_{U_{1} \times U_{2}}(n, n)$ by Definition 11. Also, it can be show that $(k, k) \npreceq U_{1} \times U_{2}(t, t)$.

Remark 1 Let $L_{1}$ and $L_{2}$ be bounded lattices, $U_{1}$ be a uninorm on $L_{1}$ with neutral element $e_{1}$ and $U_{2}$ be a uninorm on $L_{2}$ with neutral element $e_{2}$ and consider their direct product $U_{1} \times U_{2}$ on $L_{1} \times L_{2}$. Then,

$$
(x, y) \preceq_{U_{1} \times U_{2}}(z, t) \Rightarrow x \leq_{1} \text { zand } y \leq_{2} t
$$

for all $(x, y),(z, t) \in L_{1} \times L_{2}$. 
Table 2: The uninorm $U_{2}$ on $L_{1}=L_{2}$

\begin{tabular}{c|ccccccccc}
\hline$U_{2}$ & 0 & $m$ & $n$ & $e$ & $p$ & $s$ & $k$ & $t$ & 1 \\
\hline 0 & 0 & 0 & 0 & 0 & $p$ & $s$ & $k$ & $t$ & 1 \\
$m$ & 0 & $m$ & $m$ & $m$ & $p$ & $s$ & $k$ & $t$ & 1 \\
$n$ & 0 & $m$ & $n$ & $n$ & $p$ & $s$ & $k$ & $t$ & 1 \\
$e$ & 0 & $m$ & $n$ & $e$ & $p$ & $s$ & $k$ & $t$ & 1 \\
$p$ & $p$ & $p$ & $p$ & $p$ & $p$ & $s$ & $k$ & $t$ & 1 \\
$s$ & $s$ & $s$ & $s$ & $s$ & $s$ & $s$ & $k$ & $t$ & 1 \\
$k$ & $k$ & $k$ & $k$ & $k$ & $k$ & $k$ & $k$ & $t$ & 1 \\
$t$ & $t$ & $t$ & $t$ & $t$ & $t$ & $t$ & $t$ & $t$ & 1 \\
1 & 1 & 1 & 1 & 1 & 1 & 1 & 1 & 1 & 1 \\
\hline
\end{tabular}

Lemma 1 Let $L_{1}$ and $L_{2}$ be bounded lattices, $T_{1}$ be a tnorm on $L_{1}$ and $T_{2}$ be a t-norm on $L_{2}$ and consider their direct product $T_{1} \times T_{2}$ on $L_{1} \times L_{2} . T_{1} \times T_{2}$ is divisible if and only if $T_{1}$ and $T_{2}$ are divisible.

Lemma 2 Let $L_{1}$ and $L_{2}$ be bounded lattices, $S_{1}$ be a $t$-conorm on $L_{1}$ and $S_{2}$ be a t-conorm on $L_{2}$ and consider their direct product $S_{1} \times S_{2}$ on $L_{1} \times L_{2} . S_{1} \times S_{2}$ is divisible if and only if $S_{1}$ and $S_{2}$ are divisible.

Proposition 5 Let $L_{1}$ and $L_{2}$ be bounded lattices, $U_{1}$ and $U_{2}$ be uninorms on $L_{1}$ and $L_{2}$ with neutral elements $e_{1}$ and $e_{2}$, respectively, $T_{1}$ and $T_{2}$ be $t$-norms on $\left[0, e_{1}\right]$ and $\left[0, e_{2}\right]$, respectively and $S_{1}$ and $S_{2}$ be t-conorms on $\left[e_{1}, 1\right]$ and $\left[e_{2}, 1\right]$, respectively. Consider direct products $U_{1} \times U_{2}$ on $L_{1} \times L_{2}, T_{1} \times T_{2}$ on $\left[0, e_{1}\right] \times\left[0, e_{2}\right]$ and $S_{1} \times S_{2}$ on $\left[e_{1}, 1\right] \times\left[e_{2}, 1\right]$. Then, $T_{1} \times T_{2}$ and $S_{1} \times S_{2}$ are divisible if and only if $\preceq_{U_{1} \times U_{2}}=\leq$.

Proposition 6 [14] Let $T_{1}$ and $T_{2}$ be t-norms on $[0,1]$ and their direct product $T_{1} \times T_{2}$ on $[0,1]^{2} . T_{1} \times T_{2}$ is divisible if and only if $T_{1} \times T_{2}$ is continuous.

Proposition 7 [14] Let $S_{1}$ and $S_{2}$ be t-conorms on $[0,1]$ and their direct product $S_{1} \times S_{2}$ on $[0,1]^{2} . S_{1} \times S_{2}$ is divisible if and only if $S_{1} \times S_{2}$ is continuous.

Corollary 1 Let $U_{1}$ and $U_{2}$ be uninorms on $[0,1]$ with neutral elements $e_{1}$ and $e_{2}$, respectively, $T_{1}$ and $T_{2}$ be t-norms on $\left[0, e_{1}\right]$ and $\left[0, e_{2}\right]$, respectively and $S_{1}$ and $S_{2}$ be t-conorms on $\left[e_{1}, 1\right]$ and $\left[e_{2}, 1\right]$, respectively. Consider direct products $U_{1} \times U_{2}$ on $L_{1} \times L_{2}$, $T_{1} \times T_{2}$ on $\left[0, e_{1}\right] \times\left[0, e_{2}\right]$ and $S_{1} \times S_{2}$ on $\left[e_{1}, 1\right] \times\left[e_{2}, 1\right]$. Then, $T_{1} \times T_{2}$ and $S_{1} \times S_{2}$ are continuous if and only if $\preceq_{U_{1} \times U_{2}}=\leq$.

\section{Some investigations on the set of comparable and incomparable elements with respect to the $\preceq_{U}$-partial order}

In this section, we investigate some properties of direct product of uninorms on bounded lattice. We define comparable and incomparable elements with respect to the $U$ partial order on bounded lattice. By using these definitions, we obtain some interesting results for direct product of uninorms on $[0,1]^{2}$.

Definition 12 Let $L$ be a bounded lattice and $U$ be a uninorm on bounded lattice $L$. The set $C_{U}$ is defined as follows:

$$
\begin{gathered}
C_{U}=\left\{x \in L \mid \text { there } \text { exist } y, y^{\prime} \in L \backslash\{0, x, 1\},\right. \\
\left.x \preceq_{U} y \text { and } y^{\prime} \preceq_{U} x\right\}
\end{gathered}
$$

It is clear that $\{0,1\} \notin C_{U}$.

Example 4 Consider the uninorm $\overline{U_{\frac{1}{4}}}:[0,1]^{2} \rightarrow[0,1]$ with neutral element $\frac{1}{4}$ defined by

$$
\overline{U_{\frac{1}{4}}}(x, y)= \begin{cases}\min (x, y) & (x, y) \in\left[0, \frac{1}{4}\right]^{2}, \\ 1 & (x, y) \in\left(\frac{1}{4}, 1\right]^{2}, \\ \max (x, y) & \text { otherwise. }\end{cases}
$$

Then, $C_{\overline{U_{\frac{1}{4}}}}=\left(0, \frac{1}{4}\right]$.

Proposition 8 Let $L_{1}$ and $L_{2}$ be bounded lattices, $U_{1}$ be a uninorm on $L_{1}$ with neutral element $e_{1}$ and $U_{2}$ be a uninorm on $L_{2}$ with neutral element $e_{2}$. If $\preceq_{U_{1}} \subseteq \preceq_{U_{2}}$, then $C_{U_{1}} \subseteq C_{U_{2}}$.

Corollary 2 Let $L_{1}$ and $L_{2}$ be bounded lattices, $U_{1}$ be a uninorm on $L_{1}$ with neutral element $e_{1}$ and $U_{2}$ be a uninorm on $L_{2}$ with neutral element $e_{2}$. If $\preceq_{U_{1}}=\preceq_{U_{2}}$, then $C_{U_{1}}=C_{U_{2}}$.

Example 5 Consider the uninorm $U_{\frac{1}{2}}:[0,1]^{2} \rightarrow[0,1]$ with neutral element $\frac{1}{2}$ defined by

$$
\underline{U_{\frac{1}{2}}}(x, y)= \begin{cases}0 & (x, y) \in\left[0, \frac{1}{2}\right)^{2}, \\ \max (x, y) & (x, y) \in\left[\frac{1}{2}, 1\right]^{2}, \\ \min (x, y) & \text { otherwise. }\end{cases}
$$

and consider the uninorm $U:=U_{\min \left(T^{n M}, S_{M}, \frac{1}{2}\right)}$ : $[0,1]^{2} \rightarrow[0,1]$ with neutral element $\frac{1}{2}$ defined as follows:

$U=U_{\min \left(T^{n M}, S_{M}, \frac{1}{2}\right)}(x, y)= \begin{cases}0 & (x, y) \in\left[0, \frac{1}{2}\right]^{2}, \\ & \text { and } x+y \leq \frac{1}{2}, \\ \max (x, y) & (x, y) \in\left[\frac{1}{2}, 1\right]^{2}, \\ \min (x, y) & \text { otherwise. }\end{cases}$ 
It can be shown that $C_{U}=\left[\frac{1}{2}, 1\right)$ and $C_{U_{\frac{1}{2}}}=\left[\frac{1}{2}, 1\right)$. That is, $C_{U}=C_{U_{\frac{1}{2}}}$. Since $\frac{1}{5} \preceq_{U} \frac{1}{3}$ and $\frac{\frac{1}{1}}{5} \npreceq_{U_{\frac{1}{2}}} \frac{1}{3}$, it does not need to $\overline{b e} \preceq_{U}=\preceq_{U_{\frac{1}{2}}}$.

The set $C_{U}$ allows us to introduce the next equivalence relation on the class of all uninorms on bounded lattices.

Definition 13 Define a relation $\delta$ on the class of all uninorms on bounded lattices by $U_{1} \delta U_{2}$

$$
U_{1} \delta U_{2}: \Leftrightarrow C_{U_{1}}=C_{U_{2}}
$$

Lemma 3 The relation $\delta$ given in Definition 13 is an equivalence relation.

Definition 14 For a given uninorm $U$ on bounded lattice $L$, we denote by $\bar{U}$ the $\delta$ equivalence class linked to $U$, i.e.

$$
\bar{U}=\left\{U^{\prime} \mid \quad U^{\prime} \delta U\right\} .
$$

If we take $L=[0,1]$, then we obtain the following Proposition 9 and Proposition 10.

Proposition 9 The set $[0,1] / \delta$ of all equivalence classes of all uninorms on the unit interval $[0,1]$ under $\delta$, is uncountably infinite.

Proposition 10 Let $e \in[0,1]$. If $U \in \mathscr{U}(e)$, then

$$
U(x, y)= \begin{cases}T_{U}(x, y) & (x, y) \in[0, e]^{2}, \\ S_{U}(x, y) & (x, y) \in[e, 1]^{2}, \\ D(x, y) & (x, y) \in A(e),\end{cases}
$$

where $T_{U}$ is a t-norm on $[0, e], S_{U}$ is a t-conorm on $[e, 1]$ and $D: A(e) \rightarrow[0,1]$ is increasing and fulfills

$\min (x, y) \leq D(x, y) \leq \max (x, y)$ for $(x, y) \in A(e)$ by [15].

If $T_{U}$ and $S_{U}$ are continuous t-norm and t-conorm, respectively, then $C_{U}=(0,1)$.

Example 6 Let $e \in[0,1]$. Consider the uninorms $U^{\min }$ and $U^{\max }$ as unique idempotent uninorm $U_{e}^{\min }$ and $U_{e}^{\max }$, respectively:

$$
\begin{aligned}
& U^{\min }(x, y)= \begin{cases}\max (x, y) & (x, y) \in[e, 1]^{2}, \\
\min (x, y) & \text { otherwise. }\end{cases} \\
& U^{\max }(x, y)= \begin{cases}\min (x, y) & (x, y) \in[0, e]^{2}, \\
\max (x, y) & \text { otherwise. }\end{cases}
\end{aligned}
$$

Then, it is obtained that $C_{U^{\min }}=(0,1)$ and $C_{U^{\max }}=$ $(0,1)$.
The next example shows the importance of continuity in Proposition 10.

Example 7 Consider the uninorm $U:=$ $U_{\min \left(T^{n M}, S_{M}, \frac{1}{2}\right)}:[0,1]^{2} \rightarrow[0,1]$ with neutral element $\frac{1}{2}$ defined in Example 5. Since $T^{n M}$ is left continous $t$-norm, it need not be $C_{U}=(0,1)$. Also, it is clear that $C_{U}=\left[\frac{1}{2}, 1\right)$.

Remark 2 We use the notation $K_{U}$ denote the set of all elements from $[0,1]$ admitting some incomparability with respect to $\preceq_{U}$. Note that any element $x \in K_{U}$ need not be incomparable to every element $y \in[0,1] \backslash\{0,1\}$. Considering the definition of $K_{U}$, it is easily seen that it is sufficient for an $x$ to be incomparable to only one element $y$ in order to be an element of $K_{U}$. So, we obtain different results for the sets $C_{U}$ and $K_{U}$ in Proposition 11 and Proposition 12, respectively.

Proposition 11 Let $L_{1}$ and $L_{2}$ be bounded lattices, $U_{1}$ be a uninorm on $L_{1}$ with neutral element $e_{1}$ and $U_{2}$ be a uninorm on $L_{2}$ with neutral element $e_{2}$ and consider their direct product $U_{1} \times U_{2}$ on $L_{1} \times L_{2}$. Then,

$$
C_{U_{1} \times U_{2}}=C_{U_{1}} \times C_{U_{2}} .
$$

Proposition 12 Let $L_{1}$ and $L_{2}$ be bounded lattices, $U_{1}$ be a uninorm on $L_{1}$ with neutral element $e_{1}$ and $U_{2}$ be a uninorm on $L_{2}$ with neutral element $e_{2}$ and consider their direct product $U_{1} \times U_{2}$ on $L_{1} \times L_{2}$. Then,

$$
K_{U_{1}} \times K_{U_{2}} \subseteq K_{U_{1} \times U_{2}}
$$

Remark 3 The converse of the Proposition 12 may not be true. Here is an example illustrating such a case.

Example 8 Consider the greatest uninorm $\overline{U_{\frac{1}{2}}}$ : $[0,1]^{2} \rightarrow[0,1]$ with neutral element $\frac{1}{2}$ defined by

$$
\overline{U_{\frac{1}{2}}}(x, y)= \begin{cases}\min (x, y) & (x, y) \in\left[0, \frac{1}{2}\right]^{2}, \\ 1 & (x, y) \in\left(\frac{1}{2}, 1\right]^{2}, \\ \max (x, y) & \text { otherwise. }\end{cases}
$$

and the smallest uninorm $U_{\frac{1}{2}}:[0,1]^{2} \rightarrow[0,1]$ with neutral element $\frac{1}{2}$ defined in Example 5.

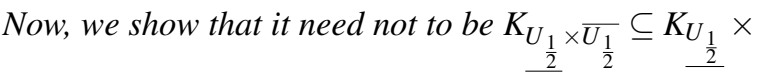
$K_{\overline{U_{1}}}$.

Remark 4 If we take the uninorms $U_{1}$ and $U_{2}$ to be equal, then the converse of the Proposition 12 is true, i.e., equality is satisfied.

Remark 5 The converse of the Proposition 12 may be true for some special uninorms on the unit interval $[0,1]$. Here is an example illustrating such a case. 
Example 9 Consider the uninorm $U_{1}:[0,1]^{2} \rightarrow[0,1]$ with neutral element $\frac{1}{2}$ defined as follows:

$U_{1}(x, y)= \begin{cases}0 & (x, y) \in\left[0, \frac{1}{2}\right]^{2} \text { and } \\ & x+y \leq \frac{1}{2} \text { and }(x, y) \neq\left(\frac{1}{4}, \frac{1}{4}\right), \\ \frac{1}{4} & (x, y)=\left(\frac{1}{4}, \frac{1}{4}\right), \\ \max (x, y) & (x, y) \in\left[\frac{1}{2}, 1\right]^{2}, \\ \min (x, y) & \text { otherwise. }\end{cases}$

and consider the uninorm $U:=U_{\min \left(T^{n M}, S_{M}, \frac{1}{2}\right)}$ : $[0,1]^{2} \rightarrow[0,1]$ with neutral element $\frac{1}{2}$ defined in Example 5. We know that $K_{U}=\left(0, \frac{1}{2}\right)$ by Aşcı (see [3]). Similarly, it can be shown that $K_{U_{1}}=\left(0, \frac{1}{2}\right)$. Also, it is clear that $K_{U \times U_{1}}=\left(0, \frac{1}{2}\right) \times\left(0, \frac{1}{2}\right)=K_{U} \times K_{U_{1}}$.

\section{Acknowledgement}

The work of the second author on this paper was supported by the grant of Slovak Research and Development Agency APVV-18-0052.

\section{Concluding remarks}

Uninorms are generalizations of $\mathrm{t}$-norms and $\mathrm{t}$ conorms with neutral element to an arbitrary point from a bounded lattice. Uninorms are useful tool in many different fields, such as expert systems, neural networks, and fuzzy logic. Moreover, they have been used as aggregators in fuzzy logic in order to maintain as many logical properties as possible. Recently, the notation of the order induced by uninorms ( $\mathrm{t}$-norms, nullnorms) has been studied widely. First, the T partial order obtained from a t-norm was defined by [27]. Based on these previous studies, the orders comprising the U-partial order and F-partial order obtained from the uninorm and nullnorm were defined by [7] and [18], respectively. The $U$-partial order is an extension of the $T$-partial order and $S$-partial order, so it is important to study the $U$-partial order to obtain more general conclusions. Thus, in this study, we have studied uninorms on bounded lattices. We have developed several new results in the domain of uninorms acting on bounded lattices, including the direct products of bounded lattices. Also, we have studied new partial orderings induced by uninorms on bounded lattices. processing, etc.

\section{References}

[1] E. Aşıc1, R. Mesiar, On generating uninorms on some special classes of bounded lattices, Fuzzy Sets and Systems, (2021) doi:10.1016/j.fss.2021.06.010.
[2] E. Aşıcı and R. Mesiar, Alternative approaches to obtain t-norms and t-conorms on bounded lattices, Iranian Journal of Fuzzy Systems 17 (2020), 121-138.

[3] E. Aş1c1, The equivalence of uninorms induced by the $U$-partial order, Hacettepe Journal of Mathematics and Statistics 48(2019) 439-450.

[4] E. Aşıc1, Equivalence classes of uninorms, Filomat 33:2 (2019) 571-582.

[5] E. Aşıc1, An extension of the ordering based on nullnorms, Kybernetika 55(2) (2019) 217-232.

[6] E. Aş1c1, On the properties of the $F$-partial order and the equivalence of nullnorms, Fuzzy Sets and Systems 346 (2018) 72-84.

[7] E. Aş1c1, An order induced by nullnorms and its properties, Fuzzy Sets and Systems 325 (2017), 35-46.

[8] E. Aşıcı, Some notes on the $F$-partial order, In: Kacprzyk J., Szmidt E., Zadroźny S., Atanassov K., Krawczak M. (eds) Advances in Fuzzy Logic and Technology 2017. IWIFSGN 2017, EUSFLAT 2017. Advances in Intelligent Systems and Computing, vol 641, pp 78-84 Springer, Cham, 2018.

[9] G. Birkhoff, Lattice Theory, 3 rd edition, Providence, 1967.

[10] T. Calvo, B. De Baets and J. Fodor, The functional equations of Frank and Alsina for uninorms and nullnorms, Fuzzy Sets and Systems 120 (2001), 385-394.

[11] J. Casasnovas and G. Mayor, Discrete t-norms and operations on extended multisets, Fuzzy Sets and Systems 159 (2008), 1165-1177.

[12] G.D. Çaylı, Uninorms on bounded lattices with the underlying t-norms and t-conorms, Fuzzy Sets and Systems 395 (2020), 107-129.

[13] G. D. Çaylı, Alternative approaches for generating uninorms on bounded lattices, Information Sciences 488 (2019) 111-139.

[14] B. De Baets and R. Mesiar, Triangular norms on product lattices, Fuzzy Sets and Systems 104 (1999), 61-75.

[15] J. Drewniak, P. Drygaś and E. Rak, Distributivity between uninorms and nullnorms, Fuzzy Sets and Systems 159 (2008), 1646-1657.

[16] D. Dubois, H. Prade, Fundamentals of Fuzzy Sets, Kluwer Acad. Publ., Boston 2000. 
[17] D. Dubois, H. Prade, A review of fuzzy set aggregation connectives, Inf. Sci. 36(1985) 85121.

[18] Ü. Ertuğrul, M.N. Kesicioğlu and F. Karaçal, Ordering based on uninorms, Information Sciences 330 (2016), 315-327.

[19] J.C. Fodor, R.R. Yager and A. Rybalov, Structure of uninorms, International Journal of Uncertainty, Fuzziness and Knowledge-Based Systems 5 (1997), 411-427.

[20] J. Fodor and B. De Baets, A single-point characterization of representable uninorms, Fuzzy Sets and Systems 202 (2012), 89âĂŞ99.

[21] M. Grabisch, J.-L. Marichal, R. Mesiar, E. Pap, Aggregation Functions, Cambridge University Press, 2009.

[22] M. Grabisch, J.-L. Marichal, R. Mesiar, E. Pap, Aggregation functions: construction methods, conjunctive, disjunctive and mixed classes, Inf. Sci. 181(2011) 23-43.

[23] M. A. İnce, F. Karaçal, R. Mesiar, Medians and nullnorms on bounded lattices, Fuzzy Sets Syst. 289 (2016), 74-81.

[24] M. Kalina, On uninorms and nullnorms on direct product of bounded lattices, Open Physics 14 (2016), 321-327.

[25] F. Karaçal and R. Mesiar, Uninorms on bounded lattices, Fuzzy Sets and Systems 261 (2015), 3343.

[26] F. Karaçal, M. A. İnce, R. Mesiar, Nullnorms on bounded lattices, Inf. Sci. 325 (2015), 227-235.

[27] F. Karaçal and M.N. Kesicioğlu, A T-partial order obtained from t-norms, Kybernetika 47 (2011), 300-314.

[28] E.P. Klement, R. Mesiar, E. Pap, Triangular Norms, Kluwer Academic Publishers, Dordrecht 2000.

[29] R. Mesiar, A. Kolesarova, A. Stupnanova Qua vadis aggregation, International Journal of General Systems 47(2) (2018) 97-117.

[30] S. Saminger, On ordinal sums of triangular norms on bounded lattices, Fuzzy Sets and Systems 157 (2006), 1403-1416.

[31] B. Schweizer, A. Sklar, Statistical metric spaces, Pacific Journal of Mathematics 10 (1960) 313-334.
[32] R.R. Yager and A. Rybalov, Uninorm aggregation operators, Fuzzy Sets and Systems 80 (1996), 111-120.

[33] R.R. Yager, Aggregation operators and fuzzy systems modeling, Fuzzy Sets and Systems 67 (1994) 129-145. 